\title{
Fanny Domenec, Catherine Resche (dir.), La Fonction argumentative de la métaphore dans les discours
} spécialisés

Berne : Peter Lang, 2018

\section{Adeline Terry}

\section{CpenEdition}

Journals

Édition électronique

URL : http://journals.openedition.org/asp/5662

DOI : $10.4000 /$ asp. 5662

ISSN : 2108-6354

\section{Éditeur}

Groupe d'étude et de recherche en anglais de spécialité

Édition imprimée

Date de publication : 2 mars 2019

Pagination : 111-115

ISSN : 1246-8185

\section{Référence électronique}

Adeline Terry, « Fanny Domenec, Catherine Resche (dir.), La Fonction argumentative de la métaphore dans les discours spécialisés », ASp [En ligne], 75 | 2019, mis en ligne le 02 mars 2019, consulté le 10 décembre 2020. URL : http://journals.openedition.org/asp/5662 ; DOI : https://doi.org/10.4000/asp. 5662

Ce document a été généré automatiquement le 10 décembre 2020.

Tous droits réservés 
Fanny Domenec, Catherine Resche (dir.), La Fonction argumentative de la métaphore dans les discours spécialisés

Berne : Peter Lang, 2018

Adeline Terry

\section{RÉFÉRENCE}

Domenec, Fanny \& Catherine Resche (dir.). 2018. La Fonction argumentative de la métaphore dans les discours spécialisés. Collection « Aspects linguistiques et culturels des discours spécialisés ». Berne : Peter Lang. 213 pages. ISSN 2296-8741. 
1 Cet ouvrage, qui traite de la fonction argumentative des métaphores dans les discours spécialisés, est le troisième volume paru dans la collection « Aspects linguistiques et culturels dans les discours spécialisés ", dirigée par Catherine Resche, Professeure à l'Université Paris 2 Panthéon-Assas. Dirigé par Fanny Domenec, maître de conférences à l'Université Paris 2 Panthéon-Assas, et Catherine Resche, il comprend huit articles en français ainsi qu'un avantpropos.

2 Le volume s'ouvre sur l'avant-propos rédigé par $\mathrm{F}$. Domenec et $\mathrm{C}$. Resche, qui retrace l'histoire des études sur la métaphore: si les propriétés rhétoriques de cette figure de style et de pensée ont

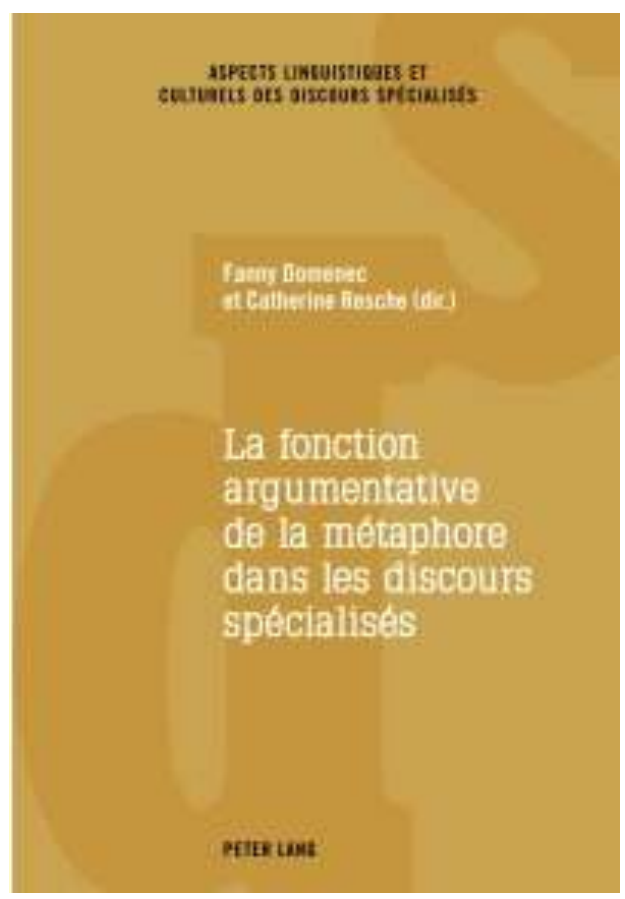
toujours été reconnues, la métaphore a longtemps été considérée avec méfiance dans les milieux non littéraires, notamment dans les milieux scientifiques. Ce n'est plus le cas aujourd'hui et cet ouvrage se propose de réconcilier ces deux aspects. En effet, les études sur la fonction argumentative de la métaphore se sont concentrées sur les discours politiques et publicitaires (Charteris-Black 2006 ; L'Hôte 2014 ; Le Guern 1981 ; Bonhomme 2009), mais assez peu sur d'autres domaines de spécialité. F. Domenec et C. Resche rappellent par ailleurs qu'il convient de faire une distinction entre discours spécialisé et discours pseudo-spécialisé (à l'instar des articles de presse). L'objectif de cet ouvrage, clairement énoncé page xi, est de «montrer que les approches liées à la fonction argumentative de la métaphore peuvent se décliner de façons diverses »; le pouvoir argumentatif de la métaphore peut être révélé par l'étude des relations entre la métaphore et la stylistique, la sémantique, l'idéologie ou la communication, la manipulation, etc.

3 Les huit articles de cet ouvrage ne sont pas organisés en sections ou chapitres, probablement parce que les domaines et les types de discours pris en compte sont différents, et que les études portent sur plusieurs langues (allemand, anglais, espagnol, français et russe), mais sont néanmoins rassemblés thématiquement. Le deuxième et le troisième article traitent de légitimation et de délégitimation par le biais de la métaphore, les trois suivants des discours portant sur des politiques économiques, et les deux derniers adoptent une approche plus multimodale.

Dans le premier article, «La métaphore biologique dans la définition de l'urbanisme moderne : nouveaux paradigmes et stratégies argumentatives autour de l'espace de la ville ", Micaela Rossi s'intéresse aux veines métaphoriques dans le langage de l'urbanisme. Elle rappelle tout d'abord que les deux veines traditionnelles pour les métaphores de l'urbanisme sont la veine organiciste et la veine mécaniciste, mais qu'elles sont loin d'être les seules. L'auteure affirme que la métaphore est un outil d'argumentation au sein du débat disciplinaire. Elle montre que dans le texte de Le Corbusier, Vers une architecture (1929), la métaphore organiciste est présentée puis 
dévalorisée pour laisser place à la métaphore mécaniciste ; cela permet à Le Corbusier de renverser la perspective métaphorique tout en conceptualisant la ville comme « un mécanisme complexe, organisé et contrôlable par l'homme " plutôt que comme « un ensemble harmonieux à l'instar du corps humain» (Rossi 2018 : 15). La métaphore permet donc d'imposer une certaine vision du réel en ayant recours à des analogies différentes. L'article s'intéresse enfin aux processus de validation de la nouvelle veine métaphorique par les autres spécialistes du même domaine. L'auteure montre avec succès que l'on peut argumenter par la métaphore et sur la métaphore.

5 Le second article, "La métaphore dans l'argumentation du Kremlin: la (dé)légitimisation des conflits ", étudie la manière dont la métaphore participe de la légitimation de la position du Kremlin tout en délégitimant l'action de l'autre dans les conflits géopolitiques dans l'argumentation du pouvoir russe. Valéry Kossov s'appuie sur un corpus de textes issus de conférences et d'interviews récentes (2014-2017) pour parvenir aux conclusions suivantes : les métaphores sont peu utilisées pour parler de situations conflictuelles dans les discours du Kremlin, ce dernier préférant éviter les images fortes dans l'argumentation. Ce choix semble indiquer que le pouvoir russe privilégie l'ethos et le logos au pathos dans ses discours. L'auteur note également des variations dans l'emploi des métaphores en fonction du public visé et du format de communication. Enfin, il conclut que les métaphores jouent un rôle argumentatif dans ces discours en ce qu'elles facilitent le passage des raisonnements à la conclusion, illustrant certains arguments, ou constituant à elles seules une conclusion.

6 Dans «Le pouvoir argumentatif de la métaphore dans le champ vexatoire : la mise en scène du monstre dans les discours propagandistes du XIXe siècle espagnol », Françoise Fournier étudie également la délégitimation de l'adversaire par le biais de la métaphore. Elle s'appuie sur deux types de textes propagandistes du XIX siècle, le libelle et le texte de genre, qui permettent tous deux de manipuler l'opinion publique, et dont la richesse rhétorique est indéniable. L'objectif de cette rhétorique étant de susciter la peur du peuple, ces deux formes de discours présentent une rhétorique du dénigrement et de la diabolisation fondée sur une vision manichéenne du monde. En étudiant la veine métaphorique de l'Autre comme monstre, l'auteure montre que les métaphores participent de cette rhétorique en créant un stéréotype social. La métaphore permet de tisser une toile autour de la cible à des fins de manipulation. En conceptualisant l'Autre comme un monstre, les propagandistes simplifient les représentations de l'Autre et légitiment leur combat pour le Bien.

7 Laurence Harris, dans « 'To change metaphor in mid-air' : paradigme et persuasion dans le discours du gouverneur de la Banque d'Angleterre à Mansion House ", adopte une approche diachronique et analyse l'évolution des veines métaphoriques dans les discours des gouverneurs de la Banque d'Angleterre en faveur de politiques monétaires entre 1946 et 2016. À travers l'analyse de deux types de veines (mécaniste et biologique ou naturelle), l'auteure montre que les métaphores utilisées par les gouverneurs suivent les paradigmes existants (à savoir le paradigme keynésien et le paradigme néolibéral) ou que, au contraire, elles semblent annoncer l'émergence d'un nouveau paradigme. Elle conclut que le fonctionnement argumentatif des métaphores du corpus repose sur deux paramètres : la familiarité et la sélection sémique. Les métaphores du corpus sont lexicalisées et surutilisées dans de nombreux domaines, ce qui les rend d'autant plus efficaces dans la stratégie argumentative, car elles imposent une vision de 
la réalité aux co-énonciateurs, qui ne perçoivent plus la réalité. Enfin, la métaphore sélectionne différents sèmes en fonction du paradigme suivi.

Le cinquième article, "La métaphore comme support argumentatif du débat économique sur les politiques d'austérité (2008-2015) ", repose sur l'étude de deux corpus : le premier reflète le discours institutionnel des banques centrales, du FMI et de la Commission européenne, et le second est composé de travaux de recherche, d'extraits de blogs et de chroniques d'économistes. Catherine Resche démontre que les partisans et les adversaires des politiques d'austérité utilisent les mêmes champs métaphoriques pour justifier leur position et attaquer l'autre camp. Ces métaphores sont également utilisées à des fins de captation; elles permettent de justifier des prises de position par le biais de la persuasion, tout en dramatisant parfois la situation. L'auteure affirme également que les métaphores servent la polémique puisqu'elles permettent de réfuter certains arguments en structurant le débat de manière partielle et partiale. De plus, les réseaux métaphoriques permettent constamment la création de nouvelles métaphores, même si certaines métaphores qui en sont issues sont figées ; pour cette raison, l'auteure suggère d'assurer une "veille métaphorique " afin de repérer les changements de position dans les discours.

Dans le sixième article, «De 'riding the ship' à 'A port of safety' : la métaphore comme argument de défense dans les lettres aux actionnaires des banques américaines (20072015) ", Fanny Domenec analyse la fonction argumentative de la métaphore dans un corpus constitué de lettres aux actionnaires publiées par Bank of America, Citi, Goldman Sachs, JPMorgan Chase, Morgan Stanley et Wells Fargo sur une période de huit ans. L'étude montre que les métaphores ont notamment été utilisées pour justifier et mettre en valeur les activités des banques après la crise des subprimes de 2008. Plus précisément, l'auteure affirme que la métaphore (et plus particulièrement la métaphore des phénomènes naturels et la métaphore mécaniste) est un argument de défense en situation de crise, et qu'elle est un outil de valorisation, notamment lorsque la métaphore de l'architecture est utilisée, ainsi que les métaphores identitaires qui permettent d'expliquer ce que la banque ne souhaite pas être, et celles qui prônent un retour à la simplicité. Enfin, la métaphore est une arme de guerre en ce qu'elle permet de critiquer le système en plus d'avoir une fonction explicative. À l'instar de l'auteure précédente, Fanny Domenec préconise de mettre en place une veille métaphorique.

Dans «Les métaphores des nanotechnologies: genres discursifs et stratégies argumentatives", Marie-Hélène Fries s'intéresse à la fonction argumentative des métaphores dans les nanotechnologies. Elle compare les métaphores utilisées dans soixante-quatre articles de recherche, des textes de vulgarisation en ligne, et trois romans de science-fiction. Ses résultats montrent que les métaphores utilisées varient en fonction du genre discursif, ainsi qu'en fonction du degré de spécialisation des textes. Les articles de recherche ont cela de spécifique qu'ils combinent une argumentation métaphorique picturale (notamment par le biais de graphiques) et une argumentation métaphorique verbale. Les textes de vulgarisation privilégient les métaphores pédagogiques car les lecteurs n'ont pas les compétences nécessaires pour comprendre les graphiques. Enfin, dans les romans de science-fiction, les métaphores participent à l'argumentation et nourrissent le récit fictionnel. En effet, ces romans étant proches d'utopies et de dystopies, ils peuvent être considérés comme des apologues - genre dans lequel se mêlent narration et argumentation. Ces métaphores participent à différentes stratégies argumentatives: le logos pour les articles de 
recherche, l'ethos pour les textes de vulgarisation et le pathos pour les romans de science-fiction.

11 Enfin, dans «La métaphore verbale et l'image matérielle dans le discours publicitaire : étude contrastive de supports publicitaires allemands, anglais et français ", Denis Jamet et Günter Schmale s'intéressent à la combinaison de métaphores verbales et d'images dans les publicités dans trois langues. Après un point théorique et un état des lieux des recherches publiées sur ce sujet, les auteurs analysent quarante-six publicités pour l'allemand, quarante-cinq pour l'anglais et vingt et une pour le français. Ils décrivent sept cas de figure: l'image matérielle peut illustrer le sens littéral de la métaphore verbale, elle peut partiellement représenter un ou plusieurs lexèmes de la métaphore verbale, la relation entre l'image et la métaphore verbale peut être implicite. Il peut ne pas y avoir de rapport transparent entre les deux éléments, le sens métaphorique de l'expression idiomatique peut être neutralisé par l'image matérielle, l'expression idiomatique peut être évoquée à travers l'image matérielle et, enfin, la métaphore verbale peut être représentée à travers l'image matérielle. Les auteurs concluent que l'argumentation publicitaire se fonde principalement la suggestion et non sur l'argumentation directe.

12 L'ouvrage propose d'étudier la métaphore dans des domaines de spécialité variés, ce qui n'a été que peu fait à notre connaissance. Il permet de porter un regard nouveau sur la fonction argumentative de la métaphore, qui ne se limite pas aux discours politiques, mais que l'on retrouve bel et bien dans plusieurs types de discours de spécialité. La richesse de l'ouvrage tient à son éclectisme, puisqu'il propose des études sur l'urbanisme, la politique, l'économie, les nanotechnologies, ou encore la publicité. La variété des articles proposés trouve une unité. En effet, l'ensemble des contributions ne manque pas de répondre aux problématiques soulevées par la fonction argumentative des métaphores dans les discours spécialisés; sont notamment explorées la question de l'utilité et de la légitimité de ces métaphores, ainsi que celle du fonctionnement pratique de la métaphore à visée argumentative. Par ailleurs, tous les articles reposent sur une étude de corpus et une méthodologie rigoureuse. Les articles se font également écho en ce que plusieurs d'entre eux ont, par exemple, recours à la terminologie aristotélicienne en faisant référence aux concepts du logos, de l'ethos et du pathos, ou encore au concept de « veines métaphoriques ».

Ainsi, cet ouvrage permet d'explorer en profondeur la dimension argumentative de la métaphore, qui a été quelque peu délaissée depuis Aristote, et notamment avec l'émergence des théories cognitivistes depuis Lakoff et Johnson (1980), qui établissent que la métaphore n'a pas uniquement une fonction ornementale. Cet ouvrage intéressera les chercheurs qui étudient les discours des domaines de spécialité de manière large, ainsi que les chercheurs qui s'intéressent à la métaphore dans la mesure où il apporte un regard nouveau, qui combine les avancées récentes dans le champ des études métaphoriques, et un retour à une approche dite "traditionnelle». Les auteurs démontrent avec succès que l'abondance de métaphores à fonction argumentative dans des discours variés entraîne la nécessité d'une veille métaphorique (Resche 2016). En résumé, l'objectif de «montrer que les approches liées à la fonction argumentative de la métaphore peuvent se décliner de façons diverses » est manifestement atteint. 


\section{BIBLIOGRAPHIE}

BONHOMME, Marc. 2009. « De l'argumentativité des figures de rhétorique ». Argumentation et Analyse du Discours 2, consulté le 23 décembre 2018. <DOI : 10.4000/aad.495>.

CHARTERIS-BLACK, Jonathan. 2006. Politicians and Rhetoric: The Persuasive Power of Metaphor. New York : Palgrave Macmillan.

LAKOFF, George \& Mark JOHNSON. 1980. Metaphors we Live By. Chicago : University of Chicago Press.

LE GUERN, Michel. 1981. « Métaphore et argumentation ». In L'Argumentation, Lyon : Presses Universitaires de Lyon, 65-74.

L'HÔTE, Émilie. 2014. Identity, Narrative, and Metaphor: A corpus-based narrative analysis of New Labour discourse. Basingtoke : Palgrave Macmillan.

RESCHE, Catherine. 2016. "Termes métaphoriques et métaphores constitutives de la théorie dans le domaine de l'économie : de la nécessité d'une veille métaphorique ». Langue française 189, 103116.

\section{AUTEURS}

ADELINE TERRY

Université Jean Moulin Lyon 3, CEL EA 1663, adeline.terry@univ-lyon3.fr 